\title{
Continuum-Level Simulations of Biopolymer Networks: A Minireview
}

\author{
Xi Wei, Pinjing Wen and Yuan Lin* \\ Department of Mechanical Engineering, The University of Hong Kong, Hong Kong, China
}

Submission: March 19, 2019; Published: April 18, 2019

*Corresponding author: Yuan Lin, Department of Mechanical Engineering, The University of Hong Kong, Hong Kong, China

\begin{abstract}
The mechanical properties of living cells are governed by the cytoskeleton, a network consisting of many different biopolymers together with transient linker proteins [1]. Biopolymers, especially those composed of globular proteins, are known to be semiflexible, that is the persistence length of these filaments is often comparable to their contour length, leading to small, yet significant, thermal fluctuations around a straight conformation and ultimately resulting in mechanical properties far from those exhibited by synthetic materials made of highly flexible polymers [2]. Theoretically, it remains a great challenge to understand/predict how a semiflexible filament network behave because of its many-body nature as well as the coupled elastic and entropic effects involved. For this reason, there has been a growing interest in the direct simulation on computer-generated networks in recent years. Here, we briefly review various continuum-level simulation approaches that have been developed in the past few decades.
\end{abstract}

\section{Mini Review}

\section{Mikado model}

(a)

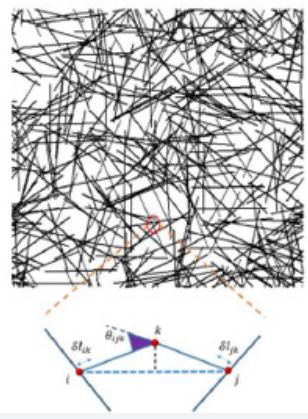

(b)

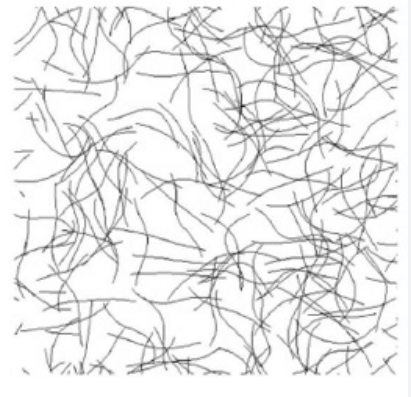

Figure 1: Illustration of (a) the Mikado model [3], and (b) an initially undulated filament network [4].

One of the simplest methods for analyzing the response of a biopolymer network is the Mikado model [2,3]. Basically, a Mikado network is constructed by randomly depositing certain number of filaments of length $l$ inside a two-dimensional square of size $W \times W$ and with periodic boundary condition, as shown in Figure 1(a). Different filaments are assumed to be permanently crosslinked together at intersection points between them. Under such circumstance, the strain energy stored in the system is expressed as

$$
H=\frac{\mu}{2} \sum\left(\frac{\delta l_{i k}^{2}}{l_{i k}}+\frac{\delta l_{j k}^{2}}{l_{j k}}\right)+\frac{k}{2} \sum \frac{\delta \theta_{i j k}^{2}}{l_{i j k}}(1)
$$

in which $i$ and $j$ correspond to two intersecting points while $k$ represents the midpoint between them. $l_{i k}$ and $l_{j k}$ stand for the length of segment $i k$ and $j k$, respectively, with $\delta l_{i k}$ and $\delta l_{j k}$ being their elongation. $l_{i j k}=\frac{1}{2}\left(l_{i k}+l_{j k}\right)$ is the average length of the two segments and $\delta \theta_{i j k}$ represents the angle change between segment $i k$ and $j k$. Essentially, this model treats the filaments as elastic rods with respect to stretching and bending, with $\mu$ and $k$ representing the corresponding stretching and bending stiffness. Interestingly, simulations from such an approach revealed that the network deformation is non-affine at small strains due to significant bending of individual biopolymers.

\section{Finite element model}

To account for the bending effect in a more realistic manner, Onck et al. [4] and Chen et al. [5] used finite element method (FEM) 
to simulate the behavior of two dimensional networks (Figure 1 b). In these models, each filament is discretized into numerous nonlinear beam elements so that the bending energy stored in the entire network can be accurately calculated. One major discovery from these studies is that the stress-stiffening of biopolymer networks is actually driven by the bending to stretching transition of filament deformation, rather than the commonly believed entropy effect. In particular, the network response was found to be dominated by filament bending at small strains. However, more filaments will be subjected to direct stretching (in the direction of straining) as strain increases which results in an elevated resistance against deformation [4], a conclusion that has also been obtained from recent simulations on 3D networks [68]. Moreover, it was shown that the addition of myosin motors, modeled as distributed force dipoles, into the network can lead to order-of magnitude increase in its apparent modulus [5]. Interestingly, recent studies also found that significant alignment and remodeling of fibrous extracellular matrices can be induced by cellular contraction, resulting in long-range force transmission and interaction among cells $[9,10]$.

\section{Combined FEM-Langevin dynamics approach}

Itis worthy to point out that thermal fluctuations of semiflexible biopolymers have not really been captured in the aforementioned continuum level simulations. The only attempt to address this was made by Onck et al. [4] and Huisman et al. [6], where an undulated shape (Figure $1 \mathrm{~b}$ ) was assigned to each filament only at the beginning to account for the thermal excitations. Nevertheless, such treatment is not very realistic as filaments should undergo fluctuations dynamically and continuously. To solve this issue, Lin et al. [11] developed a Langevin dynamics-based formulation to describe the shape undulations of biopolymers. In particular, according to Langevin [12], the effect of collisions of medium molecules on the biofilament can be represented by two terms: a viscous force $\zeta V$ acting on the biopolymer with $\zeta$ being the viscous coefficient per unit length and $V$ corresponding to the local velocity of the moving biopolymer; and a random force $f$ distributed along the filament (Figure 2a). This formulation was then implemented in the FEM simulations [11], where the transverse load $\left[\begin{array}{ll}t_{j} & t_{j+1}\end{array}\right]$, as shown in Figure 2 a, was generated randomly from a Gaussian distribution with zero mean and a variance of $2 \zeta k_{B} T / \Delta x \Delta t$, with $\Delta x$ and $\Delta t$ being the element length and the size of the time step, respectively. Using this method, simulations showed that the response of randomly cross-linked F-actin networks undergoes transitions from being entropy governed to directed by filament bending and then, to eventually dominated by filament stretching as deformation progresses [11]. (a)

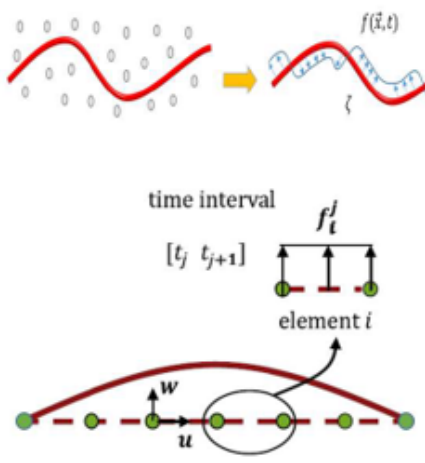

(b)

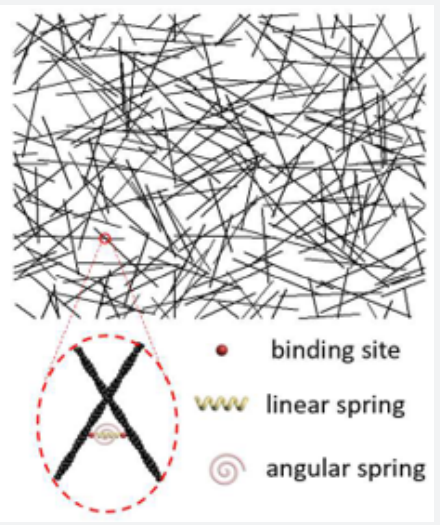

Figure 2: (a) The FEM-Langevin dynamics model [9]. A biopolymer immersed in a thermal bath undergoes continuous shape fluctuations. The effects of bombardment of medium molecules on the motion of the filament can be represented by a macroscopic medium viscosity $\xi$ and a distributed random load $f$. Within the standard FEM procedure, an un-deformed filament (dashed line) is divided into numerous beam segments and a transverse load $f_{i}^{j}$, acting on element $i$ over the time interval $\left[t_{j} t_{j+1}\right]$ is applied to represent the effect of thermal excitations (b) Filamentous network with compliant cross-linkers, modeled as a combination of linear and rotational springs [12].

Given that a wide variety of crosslinking proteins, with distinct physical properties, are involved in constructing the cytoskeleton of cells [13], the model developed in [11], has also been extended recently to examine how the deformability of cross-linkers regulates the bulk mechanical response and failure of biopolymer networks [14]. In that study, as shown in Figure 2(b), the crosslinking molecule was modeled as a combination of linear and angular springs that resist both the separation and rotation of two filaments.

In addition, the cross-linker was assumed to break once the strain energy stored inside is over a critical level. Interestingly, it was observed that the stress-strain curve of biofilament networks often shows transitions from linear increasing, to strain hardening, to stress serration and eventually to total fracture because of the interplay between the bending and stretching of individual filaments as well as the deformation and breakage of cross-linkers. Furthermore, the network fracture energy was found to reach its minimum at intermediate rotational compliance of crosslinking molecules, reflecting the fact that the imposed deformation tends to be evenly distributed among soft crosslinkers (with high rotational compliance) while most strain energy will be stored in the distorted filaments with rigid crosslinking proteins [14]. 


\section{Conclusion}

In this minireview we discussed some of the main continuumlevel simulation approaches that have been developed to study the response of semiflexible biopolymer networks in the last few decades. It must be pointed out that, besides these methods, constitutive modeling $[8,15,16]$, and Brownian dynamics-based simulations [17-19], have also been widely used in this area, refer to $[20,21]$, for a more comprehensive discussion. With help from the experimental side, it is conceivable that more biological features can be incorporated into different continuumlevel computational platforms in the future, allowing us to systematically examine important issues like the motility $[22,23]$, volume regulation $[24,25]$, and shape transformation [26,27], of cells where cytoskeleton dynamics is known to play a key role. In addition, these simulation tools can also provide insights for the development of active biomimetic materials $[28,29]$ in the future.

\section{References}

1. Pritchard RH, Huang YY, Terentjev EM (2014) Mechanics of biological networks: from the cell cytoskeleton to connective tissue. Soft Matter 10 (12): 1864-1884.

2. Wilhelm J, Frey E (2003) Elasticity of stiff polymer networks. Phys Rev Lett 91 (10): 108103.

3. Head DA, Levine AJ, Mackintosh FC (2003) Deformation of cross-linked semiflexible polymer networks. Phys Rev Lett 91(10): 108102.

4. Onck PR, Koeman T, van Dillen T, van der Giessen E (2005) Alternative explanation of stiffening in cross-linked semiflexible networks. Phys Rev Lett 95(17): 178102

5. Chen P, Shenoy VB (2011) Strain stiffening induced by molecular motors in active crosslinked biopolymer networks. Soft Matter 7(2): 355-358.

6. Huisman EM, van Dillen T, Onck PR, van der Giessen E (2007) Three dimensional cross-linked F-actin networks: relation between network architecture and mechanical behavior. Phys Rev Lett 99(20): 208103.

7. Broedersz CP, Mao X, Lubensky TC, Mackintosh FC (2011) Criticality and isostaticity in fibre networks. Nat Phys 7(12): 983-988.

8. Broedersz CP, Sheinman M, Mackintosh FC (2012) Filament-length controlled elasticity in 3D fiber networks. Phys Rev Lett 108(7): 078102.

9. Abhilash AS, Baker BM, Trappmann B, Chen CS, Shenoy VB (2014) Remodeling of fibrous extra cellular matrices by contractile cells: predictions from discrete fiber network simulations. Biophys J 107(8): $1829-1840$

10. Wang H, Abhilash AS, Chen CS, Wells RG, Shenoy VB (2014) Long range force transmission in fibrous matrices enabled by tension-driven alignment of fibers. Biophys J 107(11): 2592-2603.

11. Lin Y, Wei X, Qian J, Sze KY, Shenoy VB (2014) A combined finite element-Langevin dynamics (FEM-LD) approach for analyzing the mechanical response of bio-polymer networks. J Mech Phys Solids 62(1): 2-18.

12. Coffey WT, Kalmykov YP (2004) The Langevin Equation: with Applications to Stochastic Problems in Physics, Chemistry and Electrical Engineering. World Scientific: Singapore.

13. Kreis T, Vale R (1999) Cytoskeletal and Motor Proteins. Oxford University Press: Oxford, UK.

14. Wei X, Zhu Q, Qian J, Lin Y, Shenoy VB (2016) Response of biopolymer networks governed by the physical properties of cross-linking molecules. Soft Matter 12(9): 2537-2541.

15. Storm C, Pastore JJ, Mackintosh FC, Lubensky TC, Janmey PA (2005) Nonlinear elasticity in biological gels. Nature 435(7039): 191-194.

16. Hu B, Shenoy VB, Lin Y (2012) Buckling and enforced stretching of biofilaments. J Mech Phys Solids 60(11): 1941-1951.

17. Shimada Y, Adachi T, Inoue Y, Hojo M (2009) Coarse-grained modeling and simulation of actin filament behavior based on Brownian dynamics method. Mol Cell Biomech 6(3): 161-173.

18. Kim T, Hwang W, Kamm RD (2009) Computational Analysis of a Crosslinked Actin-like Network. Exp Mech 49(1): 91-104.

19. Gong B, Lin J, Wei X, Qian J, Lin Y (2019) Cross-linked biopolymer networks with active motors: mechanical response and intra-network transport. J Mech Phys Solids 127: 80-93.

20. Broedersz CP, MacKintosh FC (2014) Modeling semiflexible polymer networks. Rev Mod Phys 86(3): 995-1036.

21. Gong B, Wei X, Qian J, Lin Y (2019) Modeling and simulations of the dynamic behaviors of actin-based cytoskeletal networks. ACS Biomater Sci Eng DOI: 10.1021/acsbiomaterials.8b01228.

22. Lin Y (2009) Mechanics model for actin-based motility. Physical Review E 79: 021916.

23. Lin Y, Shenoy VB, Hu B, Bai L (2010) A microscopic formulation for the actin-driven motion of Listeria in curved paths. Biophys J 99(4): 1043-1052.

24. Hui TH, Zhou ZL, Qian J, Lin Y, Ngan AHW, et al. (2014) Volumetric deformation of live cells induced by pressure-activated crossmembrane ion transport. Phys Rev Lett 113(11): 118101.

25. Hui TH, Kwan KW, Chun Yip TT, Fong HW, Ngan KC, et al. (2016) Regulating the membrane transport activity and death cells via electroosmotic manipulation. Biophys J 110(12): 2769-2778.

26. Zhu Q, Zheng F, Liu AP, Qian J, Fu C, et al. (2016) Shape transformation of the nuclear envelope during closed mitosis. Biophys J 111(10): 2309-2316.

27. Yang L, Gong Z, Lin Y, Chinthapenta V, Li Q, et al. (2017) Disordered topography mediates filopodial extension and morphology of cells on stiff materials. Advanced Functional Materials 27(38): 1702689.

28. Soares e Silva M, Depken M, Stuhrmann B, Korsten M, MacKintosh FC, et al. (2011) Active multistage coarsening of actin networks driven by myosin motors. Proc Natl Acad Sci USA 108(23): 9408-9413.

29. Kasza KE, Rowat AC, Liu J, Angelini TE, Brangwynne CP, et al. (2007) The cell as a material. Curr Opin Cell Biol 19(1): 101-107. 


\section{Your next submission with Juniper Publishers} will reach you the below assets

- Quality Editorial service

- Swift Peer Review

- Reprints availability

- E-prints Service

- Manuscript Podcast for convenient understanding

- Global attainment for your research

- Manuscript accessibility in different formats ( Pdf, E-pub, Full Text, Audio)

- Unceasing customer service

Track the below URL for one-step submission https://juniperpublishers.com/online-submission.php 